\title{
A Conceptual Model of Farm Management Information System for Decision Support
}

\author{
George Burlacu ${ }^{1}$, Ruben Costa ${ }^{2}$, Joao Sarraipa ${ }^{2}$, Ricardo Jardim-Goncalves ${ }^{2}$, \\ and Dan Popescu ${ }^{1}$ \\ ${ }^{1}$ University "Politehnica" of Bucharest, \\ Faculty of Automatic Control and Computer Science, Bucharest, Romania \\ ${ }^{2}$ Centre of Technology and Systems, CTS, UNINOVA, 2829-516 Caparica, Portugal \\ \{burlacu_george85, dan_popescu_2002\}@yahoo.com, \\ \{rddc, jfss, rg\} @uninova.pt
}

\begin{abstract}
In a today economy, it is crucial to have systems able to handle information with precision. In addition, it is also important to apply technological innovations in the various domains, with the objective to modernize and transform them to become more competitive. In this paper, a conceptual framework for a Farm Management Information System (FMIS) is presented. It is focused in the different ways of using the information coming from various sources as sensors to assist farmers in decision making of agriculture business.
\end{abstract}

Keywords: Ontology, Management Information System, Data Acquisition.

\section{$1 \quad$ Introduction}

Precision agriculture is a modern method to make agriculture, which refers to optimizing the production through the fusion of traditional mechanized agriculture procedures with new technologies such as monitoring systems, command \& control systems, geographical location systems and support information systems. These optimizations have the aim of choosing the right time for culture seeding, at the right place and monitoring the culture throughout the growth period, depending on the various parameters.

Precision agriculture uses various tools for collecting information such as soil quality sampling, remote field sensing and yield monitoring. Optimization focuses on increasing yields, reducing cultivation costs and minimizing environmental impacts [1]. The challenges that precision agriculture tries to overcome are:

- Automatic collection of the related information about the physical structure and chemical composition of the soil;

- Development of a well-structured database, which should be integrated in GIS (Geographical Information Systems);

- Development of intelligent agricultural machinery for farm working with high degree of spatial accuracy;

- Nutrients distribution in variable doses required for uniform plants growth, in order to compensate the unevenness of soil characteristics; 
- Pesticides applications have to take into consideration the nature of existing pests and weeds in crops [2];

- Making crop productivity maps in real time based on flow sensors mounted in combine hopper that harvested grain [3];

- Making electrical conductivity distribution maps of soil. These maps provide an overview of water distribution and concentration in soil [4].

Traditional agriculture uses a variety of techniques to improve land quality in order to make it suitable for planting. This includes using animal manure and digging water-channels for field's irrigation. Modern agriculture uses technologies for plant breeding and pesticides and fertilizers optimization.

\section{Relationship to Collective Awareness Systems}

Collective Awareness Systems are designed to support the environment, by sharing knowledge, best practices and processes, in order to achieve changes in lifestyle, in terms of production and consumption [5].

In this context, the paper propose a framework for a Farm Management Information System, which is a system able to manage the information with precision, having the purpose to optimize both resources and processes that occurs in the farm, using sensors systems and other types of technologies for monitoring the land.

Furthermore, the paper present an ontology that has been developed with the aim of representing the knowledge used for understanding the concepts and relations regarding the Farm Management Information System. Also, the ontology offers support to farmers in decision-making regarding the land and crop management.

\section{Related Work}

A Farm Management Information System (FMIS) is a management information system designed to assist agricultural farmers to perform various tasks ranging from operational planning, implementation and documentation for assessment of performed field work.

Sørensen proposed a conceptual model to develop a FMIS (Fig. 1a)). The main actor in this picture is the farm manager who needs to manage the overall agricultural crop production. The managerial demands are caused not only by the internal farm activity of production, but also by external entities like government, customers, universities, who increase the pressure on the agricultural sector to change the methods of production, whether the quality, the price or the technological improvements is considered [6]. The proposed FMIS aims to support management tasks and real-time decision making, as well as compliance management by automating data acquisition, contextualization operations taking into account external parameters (e.g., regulations, Best Management Practices (BMP), market information, etc.). In addition, the structure of FMIS should allow the connection with external systems (e.g., market, financial, administration, etc.). 


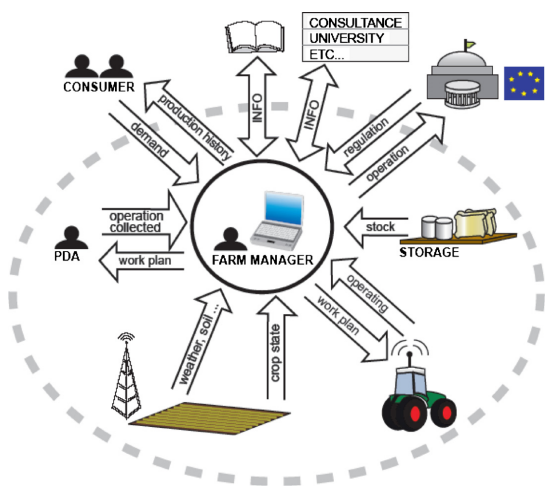

a)

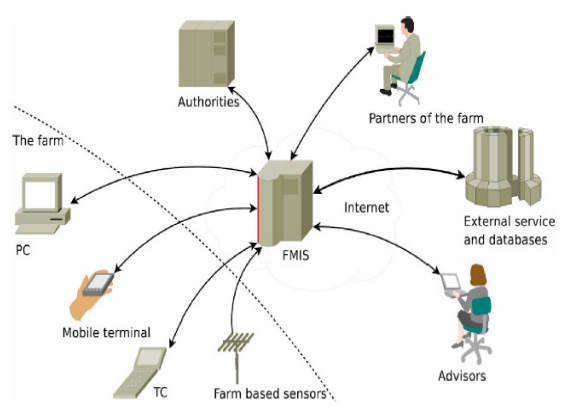

b)

Fig. 1. FMIS: a) A Conceptual model [6]; b) Architecture from the user viewpoint [7]

The automatically acquired data provide complete monitoring of operational activities. The farm manager Information initiates the both internal and external filtering, taking into account the planned operational activities. Based on this, an execution plan can be generated and sent to the executants (e.g. equipment, staff or supply service that are designed to perform the operation).

In addition to the conceptual model proposed by Sørensen, Payman Salami and Hojat Ahmadi [7] defined a system architecture (Fig. 1b)) focused in how the user of the system should understand it. The communication is represented by arrows, which are intentionally left vague, meaning that they do not have a specific protocol or content of the communication. The end user does not need to know or even care how the communication is handled between the various systems, only that it occurs and that it is possible.

In Romania, agriculture plays an important role in our daily lives; many families produce the necessary revenues existence in this way. In recent years, this sector has increasingly presented more interest and attracted many development funds. Even so, many farmers choose to work the land in the traditional way. Their motivation is mainly related to the financial aspect necessary to implement a FMIS.

\section{$4 \quad$ Farm Management Information System Framework}

The new agricultural technologies such as Variable Rate Technologies (VRT), Remote Sensing, GIS and Global Positioning System (GPS), in addition to the developments in modelling and simulation of crop production, provide numerous opportunities for the development of Precision Agriculture. In Fig. 2 is represented a new conceptual model of FMIS. Unlike the conceptual models proposed by Sørensen, Salami and Ahmadi, this framework focuses on the way it performs data acquisition, mapping, making spatial distribution maps, productivity maps and profit maps. The acquired information is stored on a platform together with the information from the various external web services; the system purposes some possibilities from its knowledge base, through which the farmer can take a decision on land or crops. 


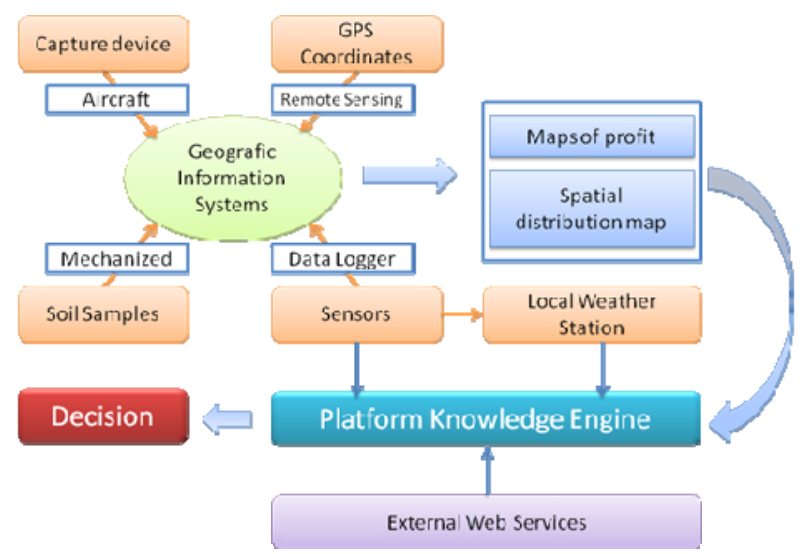

Fig. 2. A FMIS Conceptual model for decision support

The purpose of Web services is to enable communication, interoperability and data exchange between applications. Through these services the farmer has access to: new technology, resources (human, materials), information from the market (competitors, prices, forecasts), information from ministries and the European Union, etc. The GIS Software's are developed for viewing and interpreting the collected data, having special options for embedding geographic latitude and longitude. Based on this data, the farmer makes spatial distribution maps, taking into account certain parameters (location of interest points, distances between certain parts with reference to whole land areas or only to certain areas of interest) [8].

Data obtained through remote acquisition methods play an important role in building databases of geographical data within a GIS. Remote acquisition implies the existence of a capture device, attached to a platform (usually aircraft) [9]. Most of data in the current systems of agricultural information management are obtained through the interpretation of aerial photographs. Using a GIS, the farmer can analyze and interpret only digital type data. So the geographic information that does not meet this requirement must be digitized. Digitization is the process through which a physical copy of a map or a plan is converted into a digital environment using programs for objects representation in $2 \mathrm{D}$ or $3 \mathrm{D}$. Digitization can be done both by hand and by using semi-automat devices that are connected to a computer and can transmit the coordinates of a point on a 2D surface.

Topographic information from GPS have various roles: fields definition in terms of geographical boundaries, monitoring the routes of agricultural machinery in the field, to observe their position in real time, mapping the soil parameters, crops mapping, maximizing the yield per hectare and monitoring the spread of specific disease in crops. Precision spatial dimension that provides the GPS system has been manifested in the classical form of mechanized agriculture, while the development of new technologies and equipment tend to exploit to the fullest the potential acquired [10]. Presently, a large amount of data from field operations are collected by agricultural machines and transmitted using various data storage and transmission media [11]. The classical sampling, which was done manually by collecting soil samples from 
different areas of land, at random or after a specific pattern, has moved in a mechanized collection by using modern agricultural machinery. This practice is known as "sampling" [12]. Sampling is used to obtain an overview distribution of soil chemical properties and/or to check for any abnormalities in normal chemical properties of soil. Also physical sizes (soil electro conductivity, soil $\mathrm{pH}$, concentrations of nitrates, potassium, sodium) are measured directly in the field by specific laboratory values methods. Also, measuring the position of these points is recorded using GPS [13].Data from the mapping process are used to estimate the production of agricultural crops, in the entire area or specific areas of interest. In this estimation process, data from the history of agricultural land are taken into account and analyzed. A profit map can be created using inputs records in crops and outputs in harvest. So, a farmer can determine what areas of the field aren't profitable.

Farmer can also make crop productivity maps, using sensors that measure and record in real time the harvested crops volume. Various parameters like quantity per hectare and flow are measured. In this way, crop productivity maps are a valuable solution in farm management. Spatial distribution maps of the relevant parameters are ranked based on several criteria (visualization, physical support, technical type mapping, type of variables, type of representation, etc.). One advantage of using spatial distribution maps is the avoidance of the surplus or deficit in natural or mineral fertilizer (nitrogen, phosphorus, potassium, magnesium, nitrates). Automated VRT allow farmers to vary inputs, such as fertilizers, pesticides and seeding rates [14]. Varying input rates aims at either increasing yields or reducing costs, depending on the farmer's goal for the production system. Auto guidance systems, on the other hand, assist equipment operators to drive through the fields so that efforts could be focused on other important tasks. Therefore, these technological tools help to reduce redundancy, labour costs and to save operation times.

The sensors are used to determine soil properties (ground temperature/air temperature, humidity), hydric stress, and degree of crop disease (using the reflection of light on the leaves to determine their level of chlorophyll). Also, the sensors are used for measuring various parameters like electrical conductivity, high concentrations of potassium, nitrates, sodium, $\mathrm{pH}$, or can be used to measure the soil and crops properties. The need for increase the production and simultaneously the efforts for saving resources make the sensor systems the best-allied tool. Their function requires the physical storage of data, using devices known as "data loggers", which are equipped with non-volatile memory. Data transmission is done either by cable (wired) or by radio signals (wireless) [15].

Using remote sensing sensors (aerial or satellite sensors) lands maps and cultures maps are obtained. The "aerial photographer" is an optical sensor that observes the variations in soil colour, crop development and land boundaries. Images obtained by satellite or by an aircraft can provide maps of vegetative indices, which reflect also the plant health, the soil conditions or the crops status. The obtained database is more accurate (contains a large number of measured values and parameters), thereby it increases the quality of the agricultural production estimations and decisions.

In Fig. 3 different use of type of sensors that can be found in farm are presented. The role of Context allocator is to adapt the nodes behaviour in order to divide the workload based on operating or network conditions. A sensor node is a node in a 
wireless sensor network. This node gathers sensory information and communicates with other connected nodes in the network and is capable to perform some processes [16]. FMIS can use sensors in three different ways: competitive; collaborative; and individual.

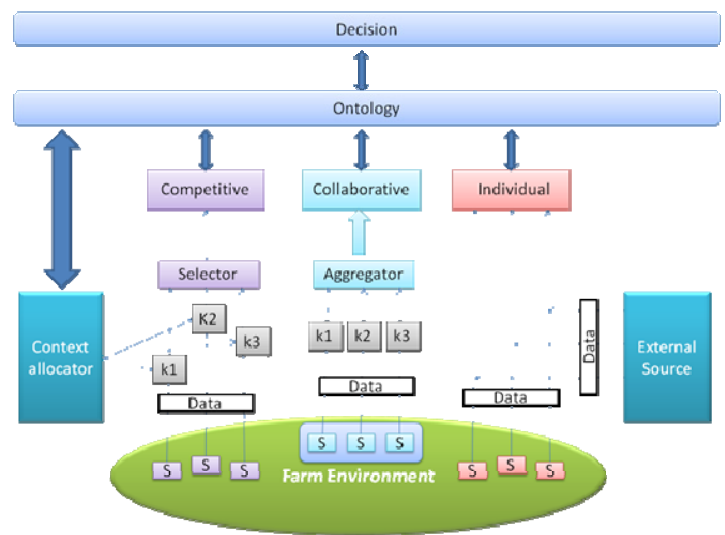

Fig. 3. Different type of sensors used in farm

The first case represents the competitive level of sensors. In this case, if the system needs to wait for the operator's intervention, maybe it becomes too slow, and thus the decision-making capability can give large benefits. So using the context allocation, new tasks are allocated to suitable nodes. In the second case, the sensors collaborate forming a network. Wireless Sensor Networks (WSNs) have been deployed as a costeffective communications technology, which allows the acquisition and transmission of different data from the crop to final users. These WSNs consists on non-intrusive communication devices of small size, to which one or more precision sensors for data collection are adapted. Once the information arrives at their operators, it is further processed and studied in order to make an appropriate decision. In the third case, using for example moisture sensors and data coming from the weather station (external source), the farmer can set a daily schedule pre-programmed for soil irrigation, according to a soil moisture level, calibrated by the farmer. A soil moisture sensor will be connected to each zone, in the relevant regions in order to assess the humidity for the entire area. Thus, the water consumption used for this purpose is minimized. If the soil moisture, at one time, is already higher than the reference level, irrigation is not done in that area and so it saves water.

\subsection{Farm Management Information System Ontology}

The ontology has been developed with the aim of representing the knowledge that can be used for understanding the concepts and relations regarding the FMIS, with the purpose of supporting farmers in decision-making regarding the land and crop management. The ontology was built through Protégé editor, using the OWL Full version, mainly because of its high expressiveness capability. Fig. 4 a) illustrates an 
excerpt of the defined ontology representing a FMIS aligned with its conceptual model (Fig. 2). The ontology intends to answer different questions about FMIS features. As an example, Fig. 4b) illustrates how the ontology represents sensors characteristics. The types of sensors used in FMIS described in [2], are represented as instances of Sensors class [1], which provides data for GIS [3], able to measure: concentration, electro-conductivity, moisture and temperature [4].

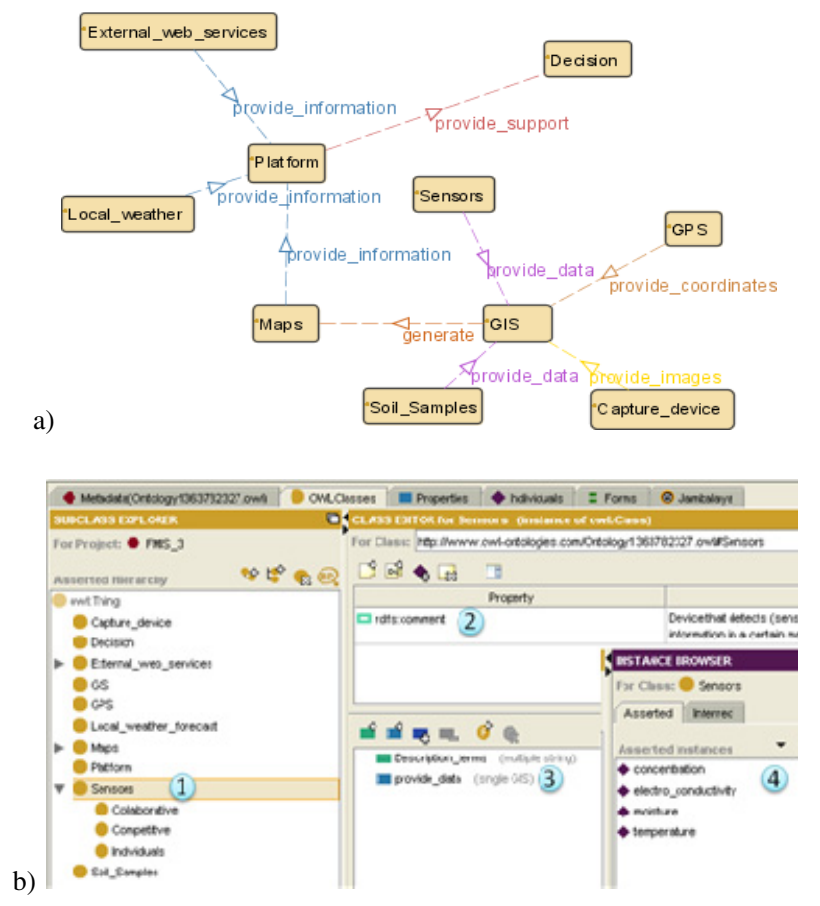

Fig. 4. FMIS Ontology: a) structure excerpt; b) sensors characterization

\section{$5 \quad$ Conclusions and Future Work}

In Romania, after many years of decline, reviving the agricultural activities has become a priority. In this context, precision agriculture supported by management information systems of the farm activities represents a viable and effective solution for its modernisation. In this paper, it is proposed a FMIS for precision agriculture, which is focused on functions to handle: land mappings, internal and external data collection, monitoring parameters, and data processing for decision support. Through such type of features, the farmer is able to see information about new technology, available resources (human, materials), information from the market (competitors, prices, forecasts), information coming from ministries and European Union, so it can take a better profitable decision. FMIS related knowledge is represented in a designed ontology to facilitate formal data acquisition from various monitoring, control, analysis and tracking systems for the referred precision farming implementation. 
In the future authors intend to develop a platform that will integrate this ontology and external services; business process modelling between farmer, suppliers and potential clients using Business Process Model and Notation (BPMN); to then effectively test the proposed FMIS in pilot farms.

Acknowledgments. This article results has been developed within the EXPERT POSDRU/107/1.5/S/76903 (http://plone.cempdi.pub.ro/BurseDoctoraleID76903) and FITMAN N 604674 (http://www.fitman-fi.eu) projects, which has been funded respectively from the European Social Fund and EC 7th Framework Programme.

\section{References}

1. Gebbers, R., Adamchuk, V.: Precision Agriculture and Food Security. In: Science, vol. 327 (2010)

2. Tellaeche, A., Artizzu, X.P.B., et al.: A new vision-based approach to differential spraying in precision agriculture. Computers and Electronics in Agriculture 60 (2008)

3. Adamchuk, V.I., Hummel, J.W., et al.: On-the-go soil sensors for precision agriculture. Computers and Electronics in Agriculture 44 (2004)

4. Johnson, C.K., Doran, J.W., et al.: Field-scale electrical conductivity mapping for delineating soil condition. Soil Science Society of America Journal 65 (2001)

5. European Commission (2013), Retrieved from the web at Dez13: http: / /ec.europa.eu/digital-agenda/en/collective-awarenessplatforms-sustainability-and-social-innovation

6. Sørensen, C., Fountas, S., Nash, E., Pesonen, L., Bochtis, D., Pedersen, S., Basso, B., Blackmore, S.: Conceptual Model of a Future Farm Management Information System. In: Computers and Electronics in Agriculture, vol. 72 (2010)

7. Salami, P., Ahmadi, H.: Review of Farm Management Information Systems (FMIS). New York Science Journal (2010)

8. Bachmaier, M., Gandorfer, M.: A conceptual framework for judging the precision agriculture hypothesis with regard to site-specific nitrogen application. Precision Agriculture 10 (2008)

9. Wang, N., Zhang, N.Q., et al.: Wireless sensors in agriculture and food industry - Recent development and future perspective. Computers and Electronics in Agriculture 50 (2006)

10. Lamba, D., Fraziera, W.P., et al.: Improving pathways to adoption: Putting the right P's in precision agriculture. Computers and Electronics in Agriculture 61 (2007)

11. Nikkilä, R.: Farm Management Information System Architecture for Precision Agriculture. Department of Computer Science and Engineering, Laboratory of Computer and Information Science. Helsinki University of technology, Espoo, Finland (2007)

12. Schnug, E., Panten, K., et al.: Sampling and nutrient recommendations - The future. In: Int. Soil and Plant Analysis Symp., Minneapolis, Minnesota, Marcel Dekker Inc. (1997)

13. Adamchuk, V.I., Morgan, M.T., et al.: An automated sampling system for measuring soil pH. Trans. Sensors (2011)

14. Srivastava, S.: Space Inputs for Precision Agriculture: Scope for Prototype Experiments in the Diverse Indian Agro-Ecosystems. In: Map Asia 2002, Report, 7 - 9, Bangkok, Thailand, 1-4 (August 2002) (accessed February 4, 2011)

15. Ruiz-Garcia, L., Lunadei, L., et al.: A Review of Wireless Sensor Technologies and Applications in Agriculture and Food Industry: SoA and Current Trends, Sensors (2009)

16. Dargie, W., Poellabauer, C.: Fundamentals of wireless sensor networks: theory and practice. John Wiley and Sons (2010) ISBN 978-0-470-99765-9 